\title{
Hemodialysis and Peritoneal Dialysis Patients' Problems: Patients' Perspective
}

\author{
Ghazanfar Rafiee ${ }^{1, *}$ \\ ${ }^{1}$ Shiraz University of Medical Sciences, Shiraz, Iran \\ Corresponding author: Shiraz University of Medical Sciences, Shiraz, Iran. Email: raf_gh2003@yahoo.com
}

Received 2021 August 02; Accepted 2021 November 17.

\begin{abstract}
Background: End-stage renal disease (ESRD) can be fatal without hemodialysis, peritoneal dialysis, or kidney transplantation. Hemodialysis and peritoneal dialysis participants confront many adverse effects due to both the disease course and the treatment program.

Objectives: This study was done to analyze hemodialysis and peritoneal dialysis patients' problems.

Methods: A qualitative exploratory study was used, and a purposeful sample of 55 hemodialysis and 47 peritoneal dialysis patients were interviewed. The data were collected through interviews. Initially, 12 open-ended questions were developed and used to stimulate discussions in interview sessions. Directed content analysis was used for the analysis of the transcribed data. After giving a code to each line or incidence, codes were then compared for similarity and differences, merged, and categorized.

Results: Themes of fatigue, diminished ability, sleeplessness, wasting time, body impairments, travel, and free-time activities limitations, low blood pressure, displeasure and gratification with hemodialysis if peritoneal dialysis patients compliant of peritoneal catheter problems, peritoneal dialysis difficulties and limitations, and gratification with peritoneal dialysis emerged.

Conclusions: Hemodialysis problems and patients' dependency on the hemodialysis machine and ward are at a high level, and patients' gratification is at a low level. Peritoneal dialysis patients, who do not get the infection, are satisfied with the dialysis method, and the patients' limitations and problems are fewer, and they are relaxed and have more freedom.
\end{abstract}

Keywords: Hemodialysis, Peritoneal Dialysis, Problems, Patient's Perspective

\section{Background}

Over 2 million people worldwide take dialysis, and this number is only a fragment of the people that conceptually would need it (1). Chronic kidney disease influences just about 7 million people in the United States, and a great number of these advance to end-stage renal disease (ESRD), which needs a transplant or some form of dialysis to support life, with a great number receiving hemodialysis (HD) (2). ESRD can be fatal without HD, peritoneal dialysis (PD), or kidney transplantation $(3,4)$. Hemodialysis patients (HDPs) confront many adverse effects due to the disease course and the treatment program (2). As a result of long-term continuous HD treatment sessions, dietary restrictions, and medication regimens, patients have to go through major changes in their lifestyles (5). The continuous ambulatory peritoneal dialysis (CAPD) technique is the most effective type of home care treatment, and it allows the patient to be more independent and active (6). CAPD technique is the most common type in Canada, the United Kingdom, Hong Kong, and Mexico (7). One of the advantages of this procedure is that the patient's vein stays intact (8). The treatments of PD may be associated with alternating and undetermined risks of fatality rate and complications (9). Contamination with environmental and skin microorganisms associated with manipulation of the transfusion set are the main causes of infection. During the past few decades, with advancement in peritoneal catheter insertion, caring for its insertion, the importance of improvement in connector technology, and the content of dialysis fluid, the result of PD has significantly improved (10). Some studies have shown a better quality of life in PD patients (PDPs) in comparison to HDPs, while other studies have shown a similar physical quality for both; nonetheless, they found higher mental quality in PDPs (11). This study analyzed and interpreted HDPs' and PDPs' problems. 


\section{Methods}

\subsection{Data Source}

A qualitative exploratory approach was used at HD and PD centers. In these centers, 345 ESRD patients were receiving dialysis (177), HD, and (168) PD. A purposive sample of 55 (30 male, 25 female) HD and 47 (20 male, 27 female) PDPs were interviewed, and their opinions about the HD and PD method, their physical and mental problems, and dialysis complications were collected through face-to-face semistructured interviews. Each interview session lasted for at least 50 minutes. Data collection and analysis proceeded concurrently, and once the themes were identified and data saturation was achieved, the interviews were discontinued. After clarifying the study objective and answering concerns verbally for each participant, informed consent was obtained from the participants. Also, ethics approval was obtained from the Research Ethics Board of the University. Initially, 12 open-ended questions related to their dialysis status, physical and mental problems before, during, and after dialysis, and dialysis modalities were used to stimulate discussions in the interview sessions.

\subsection{Data Analysis}

Directed content analysis was used for analyzing the transcribed data. Initially, information analysis was done to understand the data, and then, the codes were developed and categorized by identifying the emphasized words, phrases, themes, or concepts within the data (12). The transcripts were read line-by-line and paragraph-byparagraph to look for a significant statement and were coded. Overall, three levels of coding were selected as appropriate.

Level 1: Examining the data line by line and extracting codes taken from the interviews with subjects.

Level 2: Comparing the coded data with each other to create categories.

Level 3: Coding is the title given to the central themes from the categories (13).

After transcribing and coding, the codes were compared for similarity and differences, merged, and categorized. Table 1 shows how different codes were given to one of the themes. The measures used in the present study were to establish rigor, including "truth value," "applicability," “consistency," and "neutrality” (14).

\subsection{Ethical Considerations}

Ethics approval (Number: S982) for gathering data was obtained from the university Research Ethics Board.

\section{Results}

After the qualitative content analysis of data, the following themes were considered as the important themes, respectively:

\subsection{Patients' Problems Due to HD}

\subsubsection{A: Experiencing Fatigue}

3.1.1.1. Diminished Ability

This theme showed that HDPs mentioned sluggishness and inability that occur after dialysis. They felt tired after each HD session, and when they were walking, they felt fatigued and could not work.

One of the participants stated that "When I walk 200 300 meters, I feel tired. I get tired quickly, and I can't lift anything. When I come home due to fatigue, I need to take a rest."

\subsubsection{B: Sleeplessness}

\subsubsection{Sleep Deprivation}

Some HDPs had trouble sleeping. They complained of having problems in falling and staying asleep. Frequent awakening from night sleep and wakening up early in the morning was another sleep disturbance in these patients.

One of the participants mentioned that "I don't feel asleep until 12 o'clock midnight; then, I wake up after 2 3 hours, and I'm not able to go back to sleep again."

Another one said that "I'm restless during night sleep. On the days that I go for dialysis, I only sleep between 1-1.5 hours at night. Then, I don't sleep until morning, and I get frustrated."

\subsubsection{C: Time Wasting}

\subsubsection{Limited Time Frame}

HDPs usually have to be present for receiving treatment in the dialysis ward at a certain time. When patients go to the dialysis center, some unforeseen events may influence dialysis delays. These delays may cause distress in a person who has tried to reach the center on time. Also, performing a physical examination and taking information before dialysis are time-consuming. After starting dialysis, they have to stay connected to the dialysis machine for a long period of time.

One of the participants said that "I have been standing here for 5 hours, and this is the main reason why I feel sick. I had to wait for 5 hours. Half of my life is wasted. Every other day dialysis wastes my time.

One other participant stated that During the dialysis days, I can't do anything or meet up with my family and friends; I just sleep." 


\begin{tabular}{lcc}
\hline Table 1. Examples of Coding & \\
\hline Level 1 Codes (Meaning Unit) & Level 2 Codes (Categories) & Level 3 Codes (Theme) \\
\hline $\begin{array}{l}\text { The delay in the onset of dialysis; Visiting dialysis center three times a week; Spending at least } 4 \text { hours for } \\
\text { each dialysis session }\end{array}$ & Lack of time & Wasting time \\
\hline
\end{tabular}

\subsubsection{D: Body Impairments}

The studied HDPs were disappointed due to reducing their physical abilities. Owing to fatigue and physical limitations associated with dialysis, their useful part of the day was wasted. Because of their poor physical condition and spending most of their valuable time sleeping, they cannot participate in their favorite activities.

One participant stated that "After dialysis, I cannot do anything for $10-12 \mathrm{~h}$; my body is exhausted. I have nausea and headache, and I sleep all the time. When I go home, I feel bored and sleepy; it seems that I have fallen down from a mountain, and I am incapable of doing anything."

\subsubsection{E: Travel and Free Time Activities Limitations}

\subsubsection{Unsuitable for Journey}

Seemingly, when HDPs think about traveling, because of fatigue and loss of power, it becomes impossible for them to travel. Since it is necessary for HDPs to anticipate and coordinate everything concerning the place, this makes it too hard for them to travel.

One participant stated that "I traveled once, but the conditions for going to a trip was not possible because HD facilities were not available. When I want to go on a trip, first I forget I am on dialysis, and when I do realize my condition, I feel it's better to stay at home.”

\subsubsection{F: Low Blood Pressure}

\subsubsection{Dizziness, Apathy, and Weakness}

Our HDPs also suffered from dizziness, sluggishness, and weakness after each session. They felt dizzy when they went for a walk. According to the patients, this feeling occurred due to a reduction in blood pressure.

One of the participants stated that “Sometimes, I can't go out the day after dialysis; my blood pressure is slightly low. The next day when I get up, I feel like I'm going to fall. Sometimes, after dialysis, I am dizzy. When I am connected to the machine, my blood pressure falls."

\subsubsection{G: Displeasure and Gratification with $H D$}

The other half of the HDPs stated that they did not have any of the above-mentioned problems and were gratified with their dialysis. They had adapted themselves to their condition.

One of the HDP who was gratified with his/her dialysis stated that “I don't have any problem before, during, or af- ter dialysis. I get a good night's sleep. I don't have anxiety. I do my daily routine activities. I travel abroad."

\subsection{Patients' Problems and Concerns with PD}

\subsubsection{A: Peritoneal Catheter Problems}

\subsubsection{Peritoneal Catheter Complications}

One of the PD participants problems reported volume overload in the abdomen and pain at the end of fluid drainage from the peritoneal space. They felt these problems during the first few sessions, but then these feelings disappeared over time.

One of the participants stated that "When I perform dialysis, at the end of each session due to withdrawing mechanism, I feel pain. I feel pressure in my abdomen when there is fluid. “

\subsubsection{Peritoneal Catheter Mechanical Problems}

Despite the advances in PD technology, the risk of infection is still considered one of the major concerns for these patients.

One of the participants in this regard pointed out that "I have changed the catheter twice because it was infected. The tunnel was infected, and I had to use medication, but my condition hadn't improved. Catheter Cuff had come out."

\subsubsection{B: PD Difficulties and Limitations}

For performing PD, the patient has to designate a place and anticipate the time and suitable place for executing it. Especially those who are employed or planning to travel are faced with many restrictions. Those who want to travel need to coordinate, transfer solutions, and find a source to obtain the solution at the destination. Providing and planning solutions is a concern for PDPs, and one of the participants stated, "I feel it is difficult to go on a vacation. At first, I thought I could go and see my daughter who lives abroad, but it is hard for me."

\subsubsection{C: Gratification with PD}

At first, performing PD appeared to be a bit difficult for the participants in the study, and minor problems were encountered, but when they gradually got used to the technique, these problems were rectified, and they were more gratified with their dialysis. These participants did not 
suffer from fatigue, insomnia, daytime sleepiness, and hypotension that most HDPs were suffering from.

One participant stated that "Now it has been a month since a catheter was inserted; when I go out, I don't feel a thing, and I don't notice it. I forget that the catheter is in my abdomen. I can socialize without any limitations. I don't have any diet restrictions. Before I started dialysis, I had hypertension, but since I have been performing PD, my blood pressure is under control."

In this regard, another participant pointed out that "I don't have restrictions in my activities. Before going out, I perform my dialysis. It is better than HD. You can take care of yourself. It doesn't waste my time."

\section{Discussion}

Majority of HDPs' complaint of fatigue, diminished ability, sleeplessness, time-wasting, physical impairments, limitations in traveling and free-time activities, low blood pressure, displeasure and gratification with HD, and PDPs compliant of peritoneal catheter problems, PD difficulties and limitations, and gratification with PD due to PD.

The number of patients with end-stage kidney diseases that are being treated with renal replacement therapy by dialysis or transplantation has been growing (15). HDassociated complications depending on the blood flow rate and the rate of solute removal include hypotension, nausea, vomiting, muscle spasm, headaches, chest pain, and disequilibrium syndrome (16). One of the most common problems that HDPs frequently complained of was fatigue. Fatigue is the most common and is the severest symptom in HDPs (17). For many HDPs, on a regular basis, after each dialysis session, the feeling of fatigue occurs (18). Fatigue is one of the most frequent side effects and has been shown to be a common nuisance for HDPs (2). Patients stressed that fatigue created major disruption to daily life, especially for those who worked or had children reliant on them (9). Fatigue is an exhausting and extremely common symptom influencing $60 \%$ to $97 \%$ of patients receiving HD and is related to the undermined quality of life, cardiovascular disease, and mortality (19).

The wasting time theme suggested waiting in the ward before the commencement of dialysis session, having to go there three times a week and each time for 4 hours in the dialysis ward, and spending time to recover after dialysis. Although HDPs refer to centers based on appointments, sometimes their session does not start on time, and the amount of time they must wait is too long. These patients were unsatisfied because of a waste of time and because they had to spend more than the usual time in an unsuitable condition. Our findings suggested that HDPs seem to be upset about losing their time because of treatment.
Although HDPs were willing to travel and thought about it, they lost their motivation because of HD's loss of physical strength. Even if they had the motivation and ability to travel, they must coordinate with the HD center in their destination for continuing their treatment, and at the destination, facilities must be available to perform HD.

Although the studied HDPs were sleepy and tired after dialysis and felt weak, they could not get enough sleep, especially in the evening after the dialysis session. These patients usually suffered from the inability to sleep until late at night, frequent awakening during the night, and not being able to go back to sleep after waking up. Since PDPs are active during the dialysis process, they sleep less during the day and have a better night's sleep (20).

Some studied PDPs reported suffering from fluid drainage pain in their abdomen at the end of each session, but they mentioned that this feeling gradually disappears. Initially, the PD catheter is felt like a foreign object in the abdomen, but this feeling is gradually reduced over time. For PDPs and their health providers, clinical consequences (PD infection and mortality) and patient-reported outcomes (fatigue, adjustability with time, ability to travel, sleep, and ability to work) were the most important issues. Overall, PD infection ranked as the most important outcome (9).

Hygiene, cleanliness, and prevention of catheter contamination are critical in PD. Since not everyone can comply with these instructions or due to space limitation, location, and the proximity of PD catheters to the digestive system, some people are more susceptible to catheter contamination. Because peritoneal catheter infection is the most common and serious complication, and despite the fact that participants had been trained to observe sterility, some of the participants were concerned about catheter infection.

Although dialysate is sufficiently provided, these patients have to prepare the equipment four times a day and each time for 30-40 minutes and also exchange the dialysate; hence, performing these steps somewhat are time-consuming. Although the spent time seems reasonable, for some patients, this procedure seems too long.

Since this type of dialysis should be performed in a suitable and uncontaminated location, sustaining such an environment is not always possible; sometimes, creating such an environment during a trip or workplace is too difficult, and this is a major limitation for this type of dialysis. Another limitation for PDPs was going on a vacation. If the dialysate solution is not available in the destination, transferring a high volume of this fluid is too hard. Although unemployed and housewives did not need to carry dialysate and they could do dialysis at home, it was more difficult for patients who worked to take the fluid along 
with themselves and find a suitable place at the workplace. Since PDPs are not dependent on the availability of dialysis wards, traveling is easier for them. PDPs need a predetermined and arranged plan for medical care support during their journey. These patients may need to contact the PD center where they wish to go on a vacation. If they want to stay for a long period of time, they should send the required equipment to the destination before traveling and designate a clean space to conduct dialysis fluid exchange (21).

Mainly, the number and diversity of problems were much higher in HDPs. Since PDPs may experience infection directly, the infection seemed to be a very big problem. If during the fluid exchange the trained hygiene procedures were observed as well as with PD equipment advancement and surgery, PDPs' problems were less than HDPs. The capability to execute PD at home, be cost-effective, reduce mortality, and have a better quality of life are PD advantages (22). PDPs had a better sense of their treatment, and they believed that their treatment had less impact on their life. PDPs feel freer and are able to manage their own treatment (23). PDPs were more flexible regarding the time of dialysis (24).

\subsection{Limitations}

This study was done in some peritoneal and HD centers of our country. For gathering more data, further studies should be done in other countries to minimize bias to the best extent possible.

\subsection{Conclusions}

HD problems and patients' dependency on the HD machine and ward are more than PD. PDPs who do not get infection have a better sense of dialysis method, and the patients' limitations and problems are lesser, and they freer. Some factors are dependent on the will of patients to prevent infection in PD that must be considered, but some other parameters are uncontainable, and if patients become aware of them, they can reduce the risk factors in order to experience a better quality of life.

\section{Footnotes}

Authors' Contribution: Ghazanfar R, and Jamshid R.: Research design, data acquisition, analysis, and interpretation, drafting the manuscript, critically revising the manuscript, administrative, technical, and material support, study supervision, obtaining the final approval.

Conflict of Interests: The authors declared that they have no conflicts of interest.
Ethical Approval: Ethics approval (No: IR.sums.Rec. S982) for gathering data was obtained from the University Research Ethics Board.

Funding/Support: This study was not supported in part from anywhere.

Informed Consent: Informed consent was obtained from the participants.

\section{References}

1. Steyaert S, Holvoet E, Nagler E, Malfait S, Van Biesen W. Reporting of "dialysis adequacy" as an outcome in randomised trials conducted in adults on haemodialysis. PLoS One. 2019;14(2). e0207045. doi:10.1371/journal.pone.0207045.[PubMed:30721242].[PubMed Central: PMC6363141].

2. Williams AG, Crane PB, Kring D. Fatigue in African American women on hemodialysis. Nephrol Nurs J. 2007;34(6):610-7. quiz 618. [PubMed: 18203569].

3. Baillie J, Lankshear A. Patients' and Relatives' Experiences of Peritonitis When Using Peritoneal Dialysis. J Ren Care. 2015;41(3):177-86. doi: 10.1111/jorc.12118. [PubMed: 25727142].

4. Kefale B. Current Management of Chronic Kidney Disease: Literature Review.JOJUN. 2018;6(2). doi: 10.19080/jojun.2018.05.555684.

5. Ferrans CE, Powers M], Kasch CR. Satisfaction with health care of hemodialysis patients. Res Nurs Health. 1987;10(6):367-74. doi: 10.1002/nur.4770100604. [PubMed: 3423308].

6. Richardson MM, Paine SS, Grobert ME, Stidley CA, Gabbay E, Harford AM, et al. Satisfaction with Care of Patients on Hemodialysis. Clin J Am Soc Nephrol. 2015;10(8):1428-34. doi: 10.2215/CJN.11241114. [PubMed: 26130617]. [PubMed Central: PMC4527035].

7. Wearne N, Kilonzo K, Effa E, Davidson B, Nourse P, Ekrikpo U, et al. Continuous ambulatory peritoneal dialysis: perspectives on patient selection in low- to middle-income countries. Int J Nephrol Renovasc Dis. 2017;10:1-9. doi: 10.2147/IJNRD.S104208. [PubMed: 28115864]. [PubMed Central: PMC5221809].

8. Baillie J, Lankshear A, Featherstone K. Perspectives on peritoneal dialysis at home: implications for the management of a chronic condition. A study protocol.J Adv Nurs. 2012;68(8):1847-57. doi: 10.1111/j.13652648.2011.05907.x. [PubMed: 22211446].

9. Manera KE, Johnson DW, Craig JC, Shen JI, Ruiz L, Wang AY, et al. Patient and Caregiver Priorities for Outcomes in Peritoneal Dialysis: Multinational Nominal Group Technique Study. Clin J Am Soc Nephrol. 2019;14(1):74-83. doi: 10.2215/CJN.05380518. [PubMed: 30573659]. [PubMed Central: PMC6364541].

10. Li PK, Szeto CC, Piraino B, Bernardini J, Figueiredo AE, Gupta A, et al. Peritoneal dialysis-related infections recommendations: 2010 update. Perit Dial Int. 2010;30(4):393-423. doi: 10.3747/pdi.2010.00049. [PubMed: 20628102].

11. Liem YS, Bosch JL, Arends LR, Heijenbrok-Kal MH, Hunink MG. Quality of life assessed with the Medical Outcomes Study Short Form 36Item Health Survey of patients on renal replacement therapy: a systematic review and meta-analysis. Value Health. 2007;10(5):390-7. doi: 10.1111/j.1524-4733.2007.00193.x. [PubMed:17888104].

12. Richards L, Morse JM. Readme first for a user's guide to qualitative methods. 3rd ed. Sage; 2013.

13. Polit DF, Bernadette P. Nursing research: Principles and methods. 8th ed. Lippincott Williams \& Wilkins; 2008.

14. Guba EG, Lincoln YS. Effective evaluation: Improving the usefulness of evaluation results through responsive and naturalistic approaches. San Francisco, CA, US: Jossey-Bass; 1981.

15. Anderson NE, Calvert M, Cockwell P, Dutton M, Aiyegbusi OL, Kyte D. Using patient-reported outcome measures (PROMs) to promote qual- 
ity of care in the management of patients with established kidney disease requiring treatment with haemodialysis in the UK (PROMHD): a qualitative study protocol. BMJ Open. 2018;8(10). e021532. doi: 10.1136/bmjopen-2018-021532. [PubMed: 30373779]. [PubMed Central: PMC6224733].

16. Suet-Ching WL. The quality of life for Hong Kong dialysis patients. J Adv Nurs. 2001;35(2):218-27. doi: 10.1046/j.1365-2648.2001.01839.x. [PubMed: 11442701].

17. Jablonski A. The multidimensional characteristics of symptoms reported by patients on hemodialysis. Nephrol Nurs J. 2007;34(1):29-37. quiz 38. [PubMed: 17345690].

18. Mollaoglu M. Fatigue in People Undergoing Hemodialysis. Dial Transplant. 2009;38(6):216-20. doi:10.1002/dat.20330.

19. Jacobson J, Ju A, Baumgart A, Unruh M, O'Donoghue D, Obrador $G$, et al. Patient Perspectives on the Meaning and Impact of Fatigue in Hemodialysis: A Systematic Review and Thematic Analysis of Qualitative Studies. Am J Kidney Dis. 2019;74(2):179-92. doi: 10.1053/j.ajkd.2019.01.034. [PubMed: 30955947].
20. Eghbali M, Shahqolian N, Nazari F, Babaee S. Comparing problems of patients with chronic renal failure undergoing hemodialysis and peritoneal dialysis referring to medical university's hospitals. Iran J Nurs Midwifery Res. 2009;14(1).

21. Corr CA. "New Normal": Life on Dialysis-The First 90 Days. National Kidney Foundation; 2007. Available from: https://www.kidney.org/ sites/default/files/docs/11-10-0307_dialysistransitionbk2_oct07_ lr_bm.pdf.

22. Asif A, Merrill D, Leon C, Ellis R, Pennell P. Strategies to minimize tunneled hemodialysis catheter use. Blood Purif. 2006;24(1):90-4. doi: 10.1159/000089443. [PubMed: 16361847].

23. Chanouzas D, Ng KP, Fallouh B, Baharani J. What influences patient choice of treatment modality at the pre-dialysis stage? Nephrol Dial Transplant. 2012;27(4):1542-7. doi: 10.1093/ndt/gfr452. [PubMed: 21865216].

24. Baillie J. Perspectives on peritoneal dialysis at home: an ethnographic study [Thesis]. Cardiff University; 2013. 